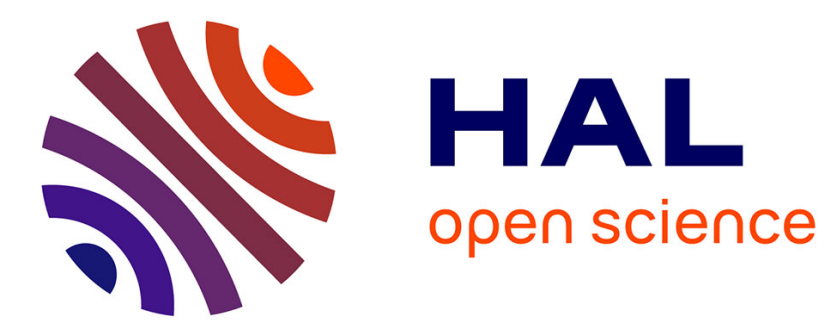

\title{
Optical properties of ZnSe-ZnTe type II superlattices
} T. Cloitre, N. Briot, O. Briot, P. Boring, B. Gil, R. Aulombard

\section{To cite this version:}

T. Cloitre, N. Briot, O. Briot, P. Boring, B. Gil, et al.. Optical properties of ZnSe-ZnTe type II superlattices. Journal de Physique IV Proceedings, 1993, 03 (C5), pp.C5-421-C5-424. 10.1051/jp4:1993590 . jpa-00251677

\section{HAL Id: jpa-00251677 https://hal.science/jpa-00251677}

Submitted on 1 Jan 1993

HAL is a multi-disciplinary open access archive for the deposit and dissemination of scientific research documents, whether they are published or not. The documents may come from teaching and research institutions in France or abroad, or from public or private research centers.
L'archive ouverte pluridisciplinaire HAL, est destinée au dépôt et à la diffusion de documents scientifiques de niveau recherche, publiés ou non, émanant des établissements d'enseignement et de recherche français ou étrangers, des laboratoires publics ou privés. 


\title{
Optical properties of $\mathrm{ZnSe}-\mathrm{ZnTe}$ type II superlattices
}

\author{
T. CLOITRE, N. BRIOT, O. BRIOT, P. BORING, B. GIL and R.L. AULOMBARD \\ Université Montpellier II, Sciences et Techniques du Languedoc, Groupe d'Etudes des \\ Semiconducteurs, case courrier 074, place E. Bataillon, 34095 Montpellier cedex 5, France
}

\begin{abstract}
In this communication we show that the cancelation of excitonic effects by photo-injected carriers can be easily produced in $\mathrm{ZnSe}-\mathrm{ZnTe}$ superlattices. This combination is interesting: it has a type II band alignment in real space. Strong confinements of electrons and holes by large bands offsets lead to important values of Rydberg energies; thus, important energy-shifts of some $20 \mathrm{meV}$ are measured when exciton screening is produced in such superlattices.
\end{abstract}

We have grown short-period superlattices by MOVPE on GaAs substrates after that either a ZnSe or a $\mathrm{ZnTe}$ buffer was deposited. The periods and thicknesses of the individual layers were obtained from $X$-ray diffraction. Collection of results obtained for a series of samples with similar designs ( 80 repeats of a $-4 \mathrm{~nm}$ building block) leads us to conclude that such superlattices are free-standing superlattices whatever the buffer is ( $\mathrm{ZnSe}$ or $\mathrm{ZnTe}$ ) [1]. To characterize the sample quality we performed both photoluminescence and transmission experiments. Results shown on figure 1 were obtained using a sample for which we deposited a 5 micrometers thick $Z n T e$ buffer layer before the 80 periods superlattice was grown. Then the substrate was removed by chemical etching using a $7 \%$ acqueous solution of $\mathrm{NaOCl}$. Figure 1 reveals the broad plateau-shaped absorption characteristics of bi-dimensional semiconductors. The shape of the absorption in type I and type II superlattices has been given in ref. 2 .

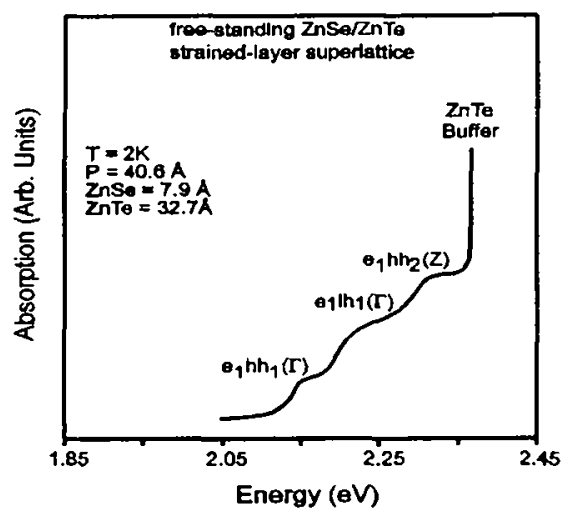

Figure 1: Optical absorption

We determine the absolute value of the absorption coefficients for the different plateaus $\left(\sim 310^{4} \mathrm{~cm}^{-1}\right)$ with an accuracy better than $25 \%$. This value is to be compared to what was obtained for other II-VI superlattices grown by MBE like $\mathrm{CdSe}-(\mathrm{Zn}, \mathrm{Mn}) \mathrm{Te}$ type II superlattices $\left(3.710^{4} \mathrm{~cm}^{-1}\right)[3]$ or $\mathrm{ZnSe}-(\mathrm{Zn}, \mathrm{Cd}) \mathrm{Se}$ type I superlattices $\left(10^{5} \mathrm{~cm}^{-1}\right)[4]$. Knowledge of the absorption coefficient is important in order to establish the relationship between the photo-injected carrier densities and the laser power. An identification of the measured transitions is offered on figure 1 after that we calculated the electronic structure of the superlattice using a multiband description of the envelope function formalism and a strain-free value of $1200 \mathrm{meV}$ for the valence band offset [5], giving an electron to heavy-hole type II configuration. The fact that the superlattice is type II has important implications on the selection rules at zone center and minizone edge. The symmetry properties of the envelope function are summarized on figure 2 and show the "allowed" character of the $e_{1} h_{2}(Z)$ transition. This transition 
associated with the miniband dispersion is the analogous of saddle-point exciton previously observed for GaAs-(Ga,Al)As and (Ga,In)As-GaAs superlattices [6]. These predictions of the envelope function approach have been completed by a calculation of the full band structure of the superlattice. The results are offered as a triptic drawing on figure 3 . In the middle part are the on-axis dispersion relations versus the superlattice wave number $Q$. Right-hand side (left-hand side) are given the in-plane dispersion relations calculated for two peculiar situations: at zone centre (minizone edge), for $Q=0(Q=\pi / L)$. The anisotropy of the in-plane dispersion relations is moderate and appears between full lines and dashed lines on the figure. We note that the on-axis dispersions relations of the first light-hole and second heavy-hole do not overlap. This is a criterion of validity of the envelope function approach: in case of overlap between these dispersions: the envelope function predicts a crossing while more elaborate tight-binding calculations predict an anticrossing (and thus a mixing) of these states [7]. Therefore the amount of mixing is small and the envelope function treatment is appropriate. Observation of sharp peak at the resonance energies is not reported. This we attribute to a broadening of the exciton resonance related to thicknesses fluctuation of both $\mathrm{ZnSe}$ and $\mathrm{ZnTe}$ layers. Though we have no evidence of the lateral size of growth terraces, we note that due to the small value of the $\mathrm{ZnSe}$ layer thickness, a monolayer thickness fluctuation has important implication on the confinement energy of the electron and is thus a good candidate to interpret a broadening of the exciton peak.
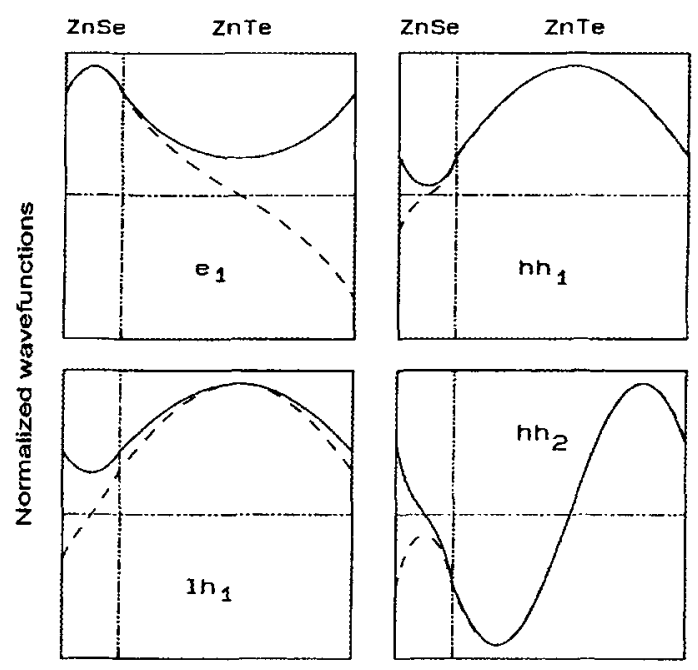

Figure 2: Typical envelope functions

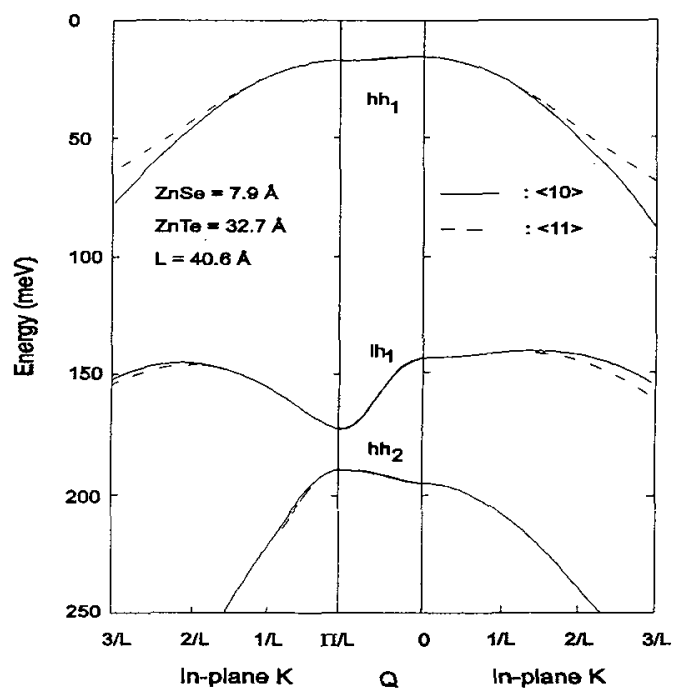

Figure 3: Full valence band structure.

We now discuss the influence of the injection density on the photoluminescence. Figure 4 displays the evolution of the photoluminescence band of the superlattice when the intensity of the $488 \mathrm{~nm}$ line of the ionized argon laser was scaling over three decades. The highest power density that we estimate from the experimental conditions is $400 \mathrm{Watt} / \mathrm{cm}^{2}$. The integrated intensity of the photoluminescence follows a linear power law of the excitation (see insert of figure 4). We note that even under strong excitation condition, we donot observe any significant broadening of the photoluminescence band, which energy saturates to a value some $25 \mathrm{meV}$ above the value measured under low excitation conditions. The magnitude of the blue-shift first depends linearly upon injection density, but saturates at higher pump intensities see figure 5), similarly with what has been observed in $\mathrm{GaAs}-(\mathrm{Ga}, \mathrm{Al})$ As microstructures of various thicknesses under moderate injection densities [8]. In our experiment, the line shape of the photoluminescence is almost independent on the photo-carrier density. Thus the saturation of the photoluminescence energy is not related to creating of a dense electron-hole plasma and subsequent onset of band gap renormalisation effects which, when occur, strongly broaden the photoluminescence and modify its lineshape. Next, we have correlated the excitation power density with the photocarrier density. Most of the incident photons $(\lambda=488 \mathrm{~nm}$ ) are absorbed in the superlattice ( the absorbtion coefficient exceeds $10^{5} \mathrm{~cm}^{-1}$ for this radiation). 




Figure 4: Photoluminescence.

In the simplest approach, neglecting diffusion and surface recombination, the injection rate writes:

$$
\begin{aligned}
& d n / d t=\left.(1-R)\right|_{0} \alpha e^{(-\alpha z)}-n / \tau=0 \\
& n=\left.(1-R)\right|_{\text {Superlattice }} ^{\alpha \tau f} e^{(-\alpha z)} d z
\end{aligned}
$$

$\mathrm{I}_{\mathrm{O}}$ represents the areal pump powerdensity, $\alpha$ and $\mathrm{R}$ are the absorption and reflectivity coefficients respectively. Thus at $400 \mathrm{watt} / \mathrm{cm}^{2}$, a plasma density of some $10^{12} \mathrm{~cm}^{-2}$ is generated if the radiative lifetime $\tau$ equals some $1 \mathrm{~ns}$, a value reasonable for type II superlattices. To calculate the induced-electric field effects correlated to the presence of free carriers in the superlattice, we have to solve local equations of the kind :

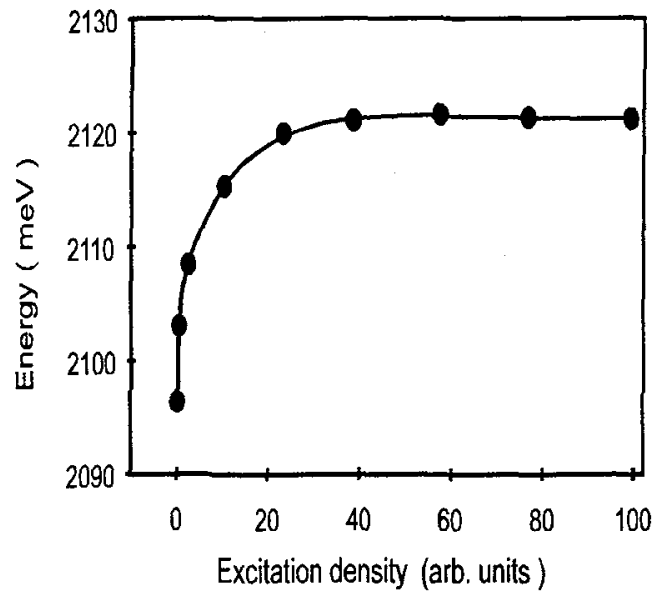

Figure 5: Experimental blue-shift.

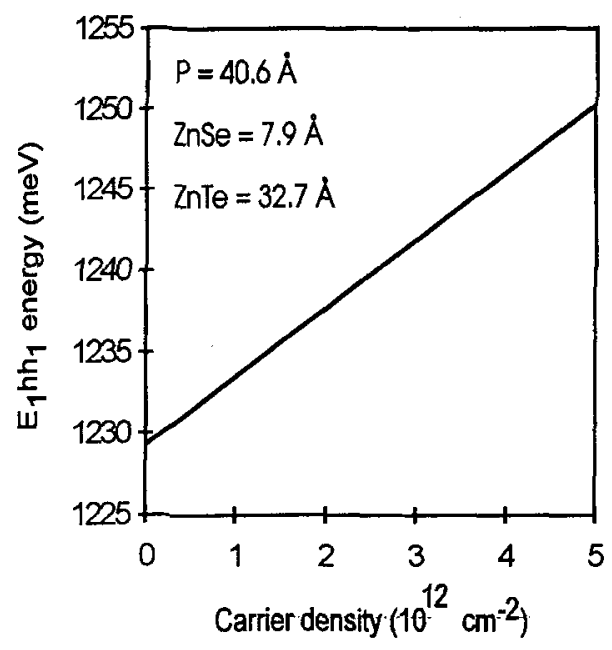

Figure 6: Theoretical blue-shift.

$$
\begin{aligned}
{\left[-h^{2} / 8 \pi^{2} \partial / \partial z 1 / m_{c}(z) \partial / \partial z+v_{c}(z)-q z \Phi-E_{C}\right] \chi_{c}(z)=0 } \\
\text { with } \Phi(z+\Delta z)-\Phi(z)=\underset{z}{z+\Delta z} \underset{z}{\sigma}\left[\left(\chi_{e}(\zeta)^{2}-\chi_{h h}(\zeta)^{2}\right) / \varepsilon(\zeta)\right] d \zeta
\end{aligned}
$$

In these equations, as usual, $\chi_{\mathcal{C}}$ represent the envelope functions, $V_{\mathcal{C}}$ are the potential line-ups and $\mathrm{m}_{c}$ represent $\mathrm{z}$-dependent carrier confining masses. The integral $\Phi$ is calculated for various areal carrier densities $\sigma$ and $\varepsilon(z)$ is the dielectric constant. $\Phi$ is nothing but the local polarisation field photo-induced between $z$ and $z+\Delta z$. We calculate a linear dependence (see figure 6), whilst the experimental observation is non-linear. At this stage we interpret the blue-shift that we observed in terms of screening 
of the exciton by photo-induced electron-hole pairs. This effect is found to be rather efficient comparing to the situation in III-V compounds. The small red-shift thus appearing on figure 5 is attributed to the onset of bandgap renormalisation [9].

\section{Acknowledgments:}

This work was supported by the Commission of the European Communities under contract Esprit III Basic Research number 6675 and by the "Région Languedoc-Roussillon".

\section{References}

[1] N. Briot, T. Cloitre, O. Briot, P. Boring, B.E. Ponga, B. Gil, R.L. Aulombard, M. Gailhanou, J.M. Sallese and A.C. Jones, to be published in proceedings of the 1993 MRS Spring Meeting (San Fransisco, CA)

[2] P. Voisin, G. Bastard, and M. Voos, Phys. Rev. B 29 (1984) 935.

[3] F.C. Zhang, H. Luo, N. Dai, N. Samarth, M. Dobrolowska, and J.K Furdyna, Phys. Rev B 47 (1993) 3806.

[4] J. Ding, N. Pelekanos, A.V. Nurmikko, H. Luo, N. Samarth, and J.K. Furdyna, Appl. Phys. Lett. 57 (1990) 2885.

[5] Y.Rajakarunanayake, R.H.Miles, G.Y.Wu and T.C.McGill, Phys.Rev.B 37 (1988) 10212.

[6] K.J. Moore, Inst. Phys. Conf. Ser. 123 (1992) 187.

[7] D.L.Smith and C.Mailhiot, Rev.Mod.Phys. 62 (1990)173.

[8] D. Hulin, A. Mysyrowicz, A. Antonetti, A. Migus, W.T. Masselink, H. Morkoç, H.M. Gibbs, and N. Peyghambarian, Phys. Rev B 33 (1986) 4389.

[9] R. Binder, I. Galbraith and S.W. Koch, Phys. Rev. B 44 (1991) 3301. 